or a few species of methylated base in the messenger. Some data also suggest that the methylated bases may be concentrated at adjacent nucleotides, perhaps located towards the $5^{\prime}$ end of the molecule; and Perry and Kelley suggest that the methylation sites are probably located in non-coding sequences of the messenger.

The proportion of methylated bases in HnRNA is much lower than in mRNA, being barely detectable. It seems possible therefore that methylation may take place in the HnRNA precursor only in those sequences destined to become mRNA, a situation analogous to the maturation of ribosomal RNA. What part this methylation plays in messenger selection must of course at present be a matter for speculation, but it is tempting to suppose that it may be involved in the selection of messenger sequences for conservation and transport to the cytoplasm.

The rationale for the evolution of the complex processing systems for both nuclear mRNA and rRNA remains elusive; it is difficult to see why half of the rRNA precursor and an even greater proportion of the HnRNA precursor should be synthesised only to be degraded. That the maturation process may be part of a system for gene control-rather than simply a feature of the mechanism of mRNA productionremains an intriguing possibility. But the presence of poly(A) in mitochondrial mRNAs throws a puzzling light on its relationship to this process, although methylation now also becomes a candidate for the role of ensuring specificity of selection. Although premature at present, it is tempting also to conclude that a general structure may be valid for all HnRNA molecules so that its features too may be important in regulating gene expression in higher cells.

\section{Controlling toxicity of chromate waste heaps}

from our Plant Ecology Correspondent

REvegetation of toxic spoil from industrial processes can be achieved only when the true nature of its toxicity has been established. This fact was ably demonstrated by Breeze's preliminary work on the chromate waste heaps of the Croal Valley in Lancashire (J.appl. Ecol., 10, 513; 1973). Gemmel (Environ. Poll., 5, 181 ; 1973) has now found that the interaction of various ions in the chemically diverse waste material of these chromate spoil heaps necessarily complicates any model of toxicity in this system.

Gemmel's technique involved the collection of samples from the waste heaps, analysing them chemically, diluting them with lime-free sand and testing their toxicity biologically using white mustard (Sinapis alba) as a test plant. In chemical analysis he found that levels of chromate, calcium, sulphate, hydroxide and bicarbonate ions were all high enough in the unweathered material to be possible causes of toxicity; in weathered waste magnesium ions also reached high concentrations.

Alleviation of toxicity before revegetation requires the determination of which of these ions is most important in the inhibition of plant growth. Gemmel attempted this by growing mustard on samples of known chemical composition and analysing the data so produced by means of correlation matrices linking dry matter production with the levels of the ions of potential interest. Highest toxicity was correlated with chromate and bicarbonate. Magnesium and calcium ions were positively correlated with dry matter production, indicating that these may alleviate the effects of the toxic ions. This is of particular interest since calcium is known to reduce the toxicity of heavy metal cations, such as lead (Wilkins, Nature, 180, 37; 1957).

If, as seems likely, toxicity is broadly determined by chromate but is modified by other ions at any given chromate level, analysis of data by simple correlation is inadequate. Gemmel tries to overcome this by grouping his samples on the basis of their chromate content together with other criteria and subsequently submitting the groups to multiple discriminant analysis to examine their validity. He then produces correlation matrices for ions and dry matter production of mustard within the groups. These indicate that hydroxide ions exert a synergistically toxic effect at medium and high chromate concentrations.

This approach, however, does not overcome the disadvantages of relying on correlations in what is evidently a chemically complex situation; for example, positive correlations between certain ions, such as magnesium and bicarbonate in weathered waste, may result from a common origin in the spoil, in this case magnesium hydroxide, reacting with carbon dioxide. If one of these ions should exert an influence on and therefore be correlated with plant growth, the other is bound to show a similar effect in terms of correlation.

As in all such multivariate situations, the ultimate solution lies in an experimental design which will allow the manipulation of the concentrations of individual ions while other ions deemed critical can be held constant. Without such an approach knowledge of the key factors controlling toxicity in waste materials must continue to be speculative.

\section{Rhythms and the Earth's rotation}

\section{from a Correspondent}

Trme is simply a progression of events, so that different clocks measure different things and their comparison gives a vital synthesis to all the sciences, even if the philosophical puzzle of the real nature of time still remains. A conference organised by G. D. Rosenberg and S. K. Runcorn on January 8-10, in the School of Physics of the University of Newcastle upon Tyne, on biological clocks and the rotation of the Earth, considered comparisons between atomic, astronomical and biological clocks and the ways in which these give information, not only on the changes in the length of the year and lunar month, but on basic organic processes, the evolution of the Solar System, changes in physical 'constants', and so on.

Several speakers (G. R. Clark, University of New Mexico; J. W. Evans, Memorial University of Newfoundland; C. Hall, University of California, Los Angles; Rosenberg; and M. A. Whyte, University of Hull) were concerned with recent experiments to determine when organisms, particularly marine bivalves and corals (R. W. Buddemeier, University of Hawaii) actually put on growth increments. It seems strange that such vital aspects have been so little studied as it is of obvious importance that some organisms show daily increments and others show additional tidal, seasonal, reproductive and circadian growth rhythms. Different animals were discussed in which one or more of these patterns could be determined, mostly by optical means, although it was sometimes necessary to resort to staining or X-ray analyses. But I. Thompson (Princeton University) showed that even when isolated from all obvious environmental stimuli, including food, some bivalves seem to regulate their biological clock with the movements of the Moon, presumably through changes in the gravity field or electromagnetic changes, so that marine organisms well below the tidal and even the photic zones may still show externally related growth rhythms.

Such fundamental findings have already become important to palaeontologists, because observations of past growth bands can determine past environments, not only on a gross scale, by the palaeolatitudinal implications of either seasonal banding or the thickness of the incrementals, but also in terms of past water depths, compositions of the substrate and local ecological relationships. The greatest interest, however, was in new results obtained by W. B. N. Berry (University of California) and G. A. L. Johnson (University of Dur- 